\title{
Push-Out Bond Strength of Composite Polymerization Methods with Universal Adhesive to Coronal Dentin
}

\author{
Horieh Moosavi $^{1}$ Fatemeh Rezaei ${ }^{2}$ Mohammad Fazli $^{3}$ Farzaneh Rakhshan ${ }^{4}$ (®)
}

${ }^{1}$ Department of Restorative and Cosmetic Dentistry, Dental Materials Research Center, School of Dentistry, Mashhad University of Medical Sciences, Mashhad, Iran

${ }^{2}$ School of Dentistry, I.M. Sechenova Medical Institute, Moscow, Russia

${ }^{3}$ Dental Material Research Center, School of Dentistry, Mashhad

University of Medical Sciences, Mashhad, Iran

${ }^{4}$ Department of Restorative and Cosmetic Dentistry, School of

Dentistry, Mashhad University of Medical Sciences, Mashhad, Iran
Address for correspondence Farzaneh Rakhshan, Department of Restorative and Cosmetic Dentistry, Mashhad University of Medical Sciences, Mashhad 9177948959, Iran (e-mail: shirin.rakhshan@gmail.com).

\begin{abstract}
Keywords

- push-out

- resin composite

- universal adhesive

Objective This in vitro study was aimed to determine the effect of composite polymerization methods with universal adhesive on push-out bond strength in coronal dentin.

Materials and Methods Using 48 healthy premolar teeth, the almost conical access cavities were excised to the canal entry. Cavity preparations were treated with a universal adhesive in the self-etch mode. Teeth were randomly divided into four groups $(n=12)$. It was used to restore the cavities with a bulk-fill composite; Tetric $N$-Ceram, a conventional composite; Filtek Z250, a dual-cure composite; Rebilda DC VOCO, and chemical cure composite; Master-Dent. After applying 10,000 thermal cycles, 1-mm incisions were made in coronal dentin, and slices were placed in a UTM machine in a special jig and tested for push-out bond strength at a crosshead speed of $0.5 \mathrm{~mm} / \mathrm{min}$. Data were analyzed using one-way analysis of variance and Games-Howell tests $(p<0.05)$.

Results The highest mean bond strength was of the conventional composite $(18.36 \pm 5.63)$ and the lowest mean of bond strength was for the dual-cure composite $(5.10 \pm 2.74)$. There was a significant difference among the means of bond strength for various composite resins curing $(p<0.001)$.

Conclusion The bulk-fill and conventional light-cured composites had higher bond strength than self- and dual-cured composite resins.
\end{abstract}

DOI https://doi.org/ $10.1055 / \mathrm{s}-0041-1736374$. ISSN 2278-9626.

\footnotetext{
(c) 2021. European Journal of General Dentistry. All rights reserved. This is an open access article published by Thieme under the terms of the Creative Commons Attribution-NonDerivative-NonCommercial-License, permitting copying and reproduction so long as the original work is given appropriate credit. Contents may not be used for commercial purposes, or adapted, remixed, transformed or built upon. (https://creativecommons.org/ licenses/by-nc-nd/4.0/)

Thieme Medical and Scientific Publishers Pvt. Ltd., A-12, 2nd Floor, Sector 2, Noida-201301 UP, India
} 


\section{Introduction}

Despite current clinical protocols that recommend the use of traditional resin composites in increments of no more than 2-mm thickness, restorative materials with fewer procedural steps and shorter working times are highly preferred by clinicians. Recent advances have led to the introduction of bulk-fill composites. ${ }^{1}$ They generally have lower filler loads and increased filler sizes to improve the depth of cure. They can also contain photoinitiators that can provide an effective depth of cure of 4-5 mm, monomers with low double-bond concentrations, and in some cases, monomers that cleave during the polymerization. Although light-cure resin composites are widely used today, chemically-activated composites still have important applications in contemporary restorative dentistry, and as a restorative material in areas that cannot be easily penetrated by light. They are also used as an auto- or dual-cure resin cement for luting crowns and bridges, inlays and on lays, as well as endodontic posts. The long pregel phase of chemical cure composites has been adopted in direct posterior resin composite restorations. ${ }^{2}$ Dual-cure composites can also further reduce shrinkage stresses. This is, in fact, the result of their low polymerization rate (due to their self-cure component) along with the delayed activation of their light-cure component (which, depending on the cure time, is activated few minutes after the start of mixing with the self-cure component). Such a delay has been shown to improve the bonding of resin cements and reduce shrinkage stress in core build-up materials and resin cements. ${ }^{3}$

Debonding takes place at the interface between the resin composite and dental substance when the existing improper bond and the shrinkage stress exceed the bond strength. Therefore, reducing the degree of polymerization shrinkage is an important factor in the development of dental resin composites. Factors that can influence shrinkage are the content of inorganic fillers and the molecular weight as well as the degree of conversion of the monomer system. ${ }^{4}$

With the increasing demand for simpler and more versatile adhesives, a new type of adhesive has emerged with which the clinician can choose the adhesion strategy and the number of steps to treat dental substrates. These so-called universal or multimode adhesives can be used in both totaland self-etch modes. However, self-cure and dual-cure resinbased materials containing basic amines are not compatible with the high concentration of acidic monomers in the simplified self-etch approach. The interaction between the monomers and the tertiary amine leads to the latter being consumed, which reduces the availability of free radicals for the polymerization reaction. ${ }^{5}$

Recent studies have used various bond strength tests, that is, macro or micro shear and tensile tests, to assess the bond strength of resin composites. In this study, however, we opted for the push-out bond strength test because it can evaluate the bond strength in cavities with a high $C$ factor (3.0) and high-stress generation directed toward the bonding area. ${ }^{4}$ The aim of this study was to compare the push-out bond strength of different composite resin curing modes (light-, self-, and dual-cure) with the universal adhesive system in self-etch mode to human coronal dentin. The null hypothesis tested was that there is no difference in the push-out bond strength of different curing modes of resin composite restorations bonded to human coronal dentin.

\section{Materials and Methods}

In this in vitro study, 60 maxillary or mandibular premolars of approximately the same size were collected. Inclusion criteria were healthy, premolar teeth, and exclusion criteria were teeth with cracks, fractures, caries, etc. The teeth were cleaned of any residual tissues stained with pumice and water and kept in Fine Tech SSI-(202) incubator in 0.1\% thymol solution at $4^{\circ} \mathrm{C}$ for 2 weeks. The roots of the teeth were removed with a Mestra $\mathrm{RH}-3000$ trimmer up to $1 \mathrm{~mm}$ below the cement enamel junction (CEJ), and almost-conical access cavities with approximately equal sizes (depth of $1 \mathrm{~mm}$ under CEJ, $3 \mathrm{~mm}$ buccolingual and mesiodistal width) were excised using a high-speed flat end Taper air/watercooled diamond milling machine. The mills were replaced after use for six teeth.

To prepare the surfaces of cavities, the universal adhesive $G$-Premio Bond was applied in the self-etch mode on the excised surface for 10 seconds using a micro brush. The surfaces were then dried with high-pressure air for 5 seconds and samples were finally cured for 10 seconds with a lightcure device (light-emitting diodes curing light CL37, 570 $\mathrm{mW} / \mathrm{cm}^{2}$ ). The cavities were randomly divided into four groups $(n=12)$, namely self-cure (Master-Dent), dual-cure (Rebilda DC), bulk-fill (Tetric $N$-Ceram), and the conventional composite (Filtek Z250). In the self-cure group, Master Dent composite with the setting and working times of 6 and 2 minutes was mixed for 20 seconds and applied incrementally in a 2-mm thickness. In the dual-cure group, Rebilda $D C$ was applied with a thickness of $2 \mathrm{~mm}$ and subsequently cured for 20 seconds. In the bulk-fill group, Tetric N-Ceram was applied in a $4-\mathrm{mm}$ thickness and cured for 20 seconds. In the conventional composite groups, Filtek Z250 was applied in a 2-mm thickness and subsequently cured for 20 seconds. Materials and their compositions used in this experiment are shown in - Table 1.

The samples were subjected to 10,000 thermal cycles in a thermocycler (Nemo Co., Mashhad, Iran). Next, 1-mm thick incisions were made from the occlusal side toward the gingival using a cutting device (Nemo Co., Mashhad, Iran). Slices without dentin on their both sides were excluded, and the remaining samples were considered for the test $(n=15)$. They further underwent the push-out test using a UTM (Santam STM-20) at a crosshead speed of $0.5 \mathrm{~mm} / \mathrm{min}$. One calibrated examiner evaluated the sections under a stereomicroscope (Dino-Lite Pro, AnMo Electronics Corp, Taiwan) at $\times 50$.

\section{Statistical Analysis}

The normality of the data was assessed using the ShapiroWilk test, and the study groups were found to have a normal 
Table 1 Materials and their compositions used in this experiment

\begin{tabular}{|l|l|}
\hline Material & Composition \\
\hline G-Premio Bond, GC Corporation, Tokyo, Japan & $\begin{array}{l}\text { 10-MPD, 4-methacryloxyethyl trimellitic anhydrate, dimethacrylate } \\
\text { monomer, distilled water, acetone, photo initiators, fine silica } \\
\text { powder (pH=1.5) }\end{array}$ \\
\hline $\begin{array}{l}\text { Tetric N-Ceram, IvoclarVivadent AG, Schaan, } \\
\text { Liechtenstein }\end{array}$ & $\begin{array}{l}\text { (Bis-GMA, UDMA, Bis-EMA), 20w\%, barium glass 63.5w\%, prepol- } \\
\text { ymer 17w\% }\end{array}$ \\
\hline Filtek Z250 (Microhybrid), 3M ESPE, St. Paul, MN, USA & Bis-GMA, UDMA, Bis-EMA, TEGDMA, (zirconia, silica) 82w\% \\
\hline Rebilda DC, VOCO, Germany & $\begin{array}{l}\text { Bis-GMA, UDMA, TEGDMA, BHT, BPO, silica, barium borosilicate } \\
\text { glass ceramic }\end{array}$ \\
\hline Master-Dent, Dentonics, USA & Catalyst paste, base paste, filler 63 w\% \\
\hline
\end{tabular}

Table 2 Mean push-out bond strengths (MPa) (SD) in each group

\begin{tabular}{|l|l|}
\hline Composite resin group & Bond strength (MPa) (SD) \\
\hline Tetric N-Ceram & $15.36(5.17)^{\mathrm{a}}$ \\
\hline Filtek Z250 & $18.36(5.63)^{\mathrm{a}}$ \\
\hline Rebilda DC & $5.10(2.74)^{\mathrm{b}}$ \\
\hline Master-Dent & $6.02(2.39)^{\mathrm{b}}$ \\
\hline
\end{tabular}

Abbreviation: SD, standard deviation.

Note: Values in parentheses are standard deviations. Superscripts indicate groups with no statistically significant difference in terms of push-out bond strengths.

distribution. One-way analysis of variance (ANOVA) was used to compare the bond strength of the groups and followed by the Games-Howell post hoc test for every possible comparison. The results of fracture patterns were submitted to Fisher's exact test. A significance level of $5 \%$ was considered in all tests.

\section{Results}

The results for the mean push-out bond strengths are shown in - Table 2. According to the results, Rebilda DC had the least mean bond strength $(5.10 \pm 2.74)$, and the mean bond strengths in the other groups were $6.02 \pm 2.39$

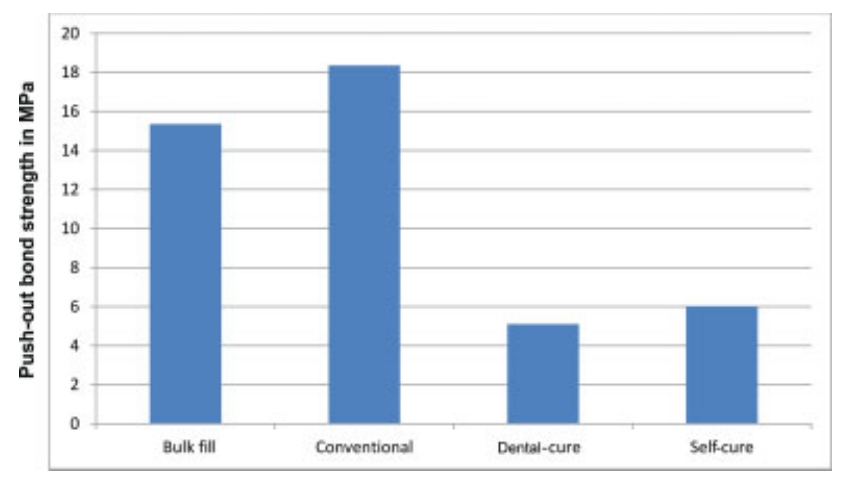

Fig. 1 Mean push-out bond strengths of resin composites.
(Master-Dent), $15.36 \pm 5.17$ (Tetric $N$-Ceram), and $18.36 \pm 5.63$ (Filtek Z250), respectively $(p<0.001)$. Moreover, Filtek Z250 had the highest mean bond strength and showed no statistically significant difference compared with Tetric N-Ceram $(p=0.440)$. The mean bond strengths in Rebilda $D C$ and Master-Dent also indicated no statistically significant difference $(p=0.763)$.

Nevertheless, the push-out bond strength in the two groups Rebilda DC and Master-Dent differed significantly with the other two groups Filtek $Z 250$ and Tetric N-Ceram $(p<0.001)$. The chart in - Fig. 1 presents the mean push-out bond strengths corresponding to the four groups.

The failure modes were classified as follows: adhesive failure between adhesive and dentin, cohesive failure in dentin, cohesive failure in resin, and mixed failure involving adhesive, dentin, and composite. The results of the classification corresponding to the four composites are shown in -Fig. 2. The four composites demonstrated a high prevalence of adhesive failures.

All fractures in the dual- and self-cure groups were adhesive fractures, whereas, in the conventional group, there were 10 cases (66.7\%) of adhesive fracture and five cases (33.3\%) of cohesive fracture in dentin. In the bulk-fill group, there were eight cases (53.3\%) of adhesive fracture, one case (6.7\%) of cohesive fracture in dentin, four cases $(26.7 \%)$ of cohesive fracture in resin composite, and two cases (13.3\%) of

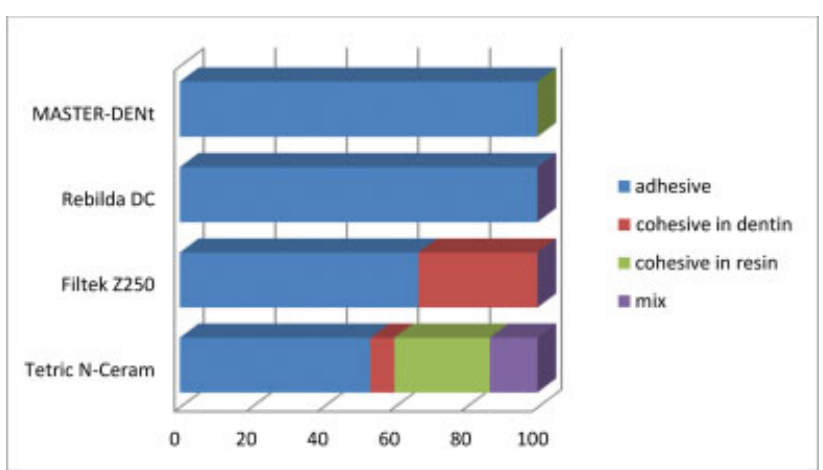

Fig. 2 Results of the analysis of the fracture modes; adhesive (adhesive failure between adhesive and dentin), cohesive in dentin (cohesive failure in dentin), cohesive in resin (cohesive failure in resin), and mixed (cohesive failure in both dentin and resin). 
mixed fracture. The distribution of fracture types in the groups was significantly different $(p<0.001)$. These results are shown in - Fig. 2.

\section{Discussion}

The null hypothesis that there was no significant difference in terms of push-out bond strengths in the examined resin composites was rejected. According to the results, the highest and lowest mean push-out bond strengths were in the conventional and dual-cure groups, respectively. Moreover, the mean push-out bond strengths in the conventional and bulk-fill groups differed significantly from those in the dualand self-cure groups.

The bond strength in the conventional group was higher than that in the bulk-fill group, although no statistically significant difference was found between the two groups, which was consistent with the study results of Mosharrafian and Sharifi (2016). ${ }^{1}$

Filtek $Z 250$ is a traditional micro-hybrid composite that has shown excellent mechanical properties in several in vitro studies. It was used in this study as a control to compare other restoration materials. Tetric $N$-Ceram bulk-fill composite is more translucent than Filtek Z250. This might result in a better depth of cure by reducing light scattering and thus improving the incident light transmission. According to the manufacturer, this resin composite has a prepolymerized shrinkage stress reliever, i.e., the photo-initiator Ivocerin (polymerization enhancer), which can polymerize the material at greater depths. It also has an organic matrix (consisting of Bis-GMA, Bis-EMA, and UDMA) that exhibits low volume shrinkage in the polymerization, as well as increased filler content of Tetric $\mathrm{N}$-Ceram bulk fill that can reduce polymerization shrinkage to some extent by increasing the filler-to-monomer ratio. ${ }^{6}$

Overall, the lower push-out bond strength in the bulk-fill composite compared with the conventional one might be due to the differences in the composition of the inorganic matrix. In the bulk-fill method, if the composite layer is relatively thick, there may not be enough double bonds for the composite to bond with the adhesive layer. Lower bond strength was also observed with shorter light-cure times. According to some studies, low radiant emittance generates a small number of free radicals, which leads to longer polymer chains with a low cross-linking density. Thus, there can be both quantitative and qualitative differences in the bond structure.

In addition to other factors such as increased filler loading, the degree of conversion and the modulus of elasticity may also influence the bond strength to the dentin., 4,8

In this study, we found that self- and dual-cured composites did not offer a good bond with universal adhesives. Recent studies have shown that some simplified-step adhesives are not compatible with self- and dual-cure composites, as implied by their reduced bond strength. ${ }^{9}$

It has been shown in a study that Prime and Bond NT, which contains PENTA (a monomer with acidic phosphate groups), did not bond to the self-cure composite if only the adhesive is combined with a sulfonic acid activator. Another study has reported that on using a self-cure composite instead of a light-cure one, the mean bond strength of the adhesive decreases by approximately 45 to $91 \% .{ }^{10}$ One factor that could contribute to this inconsistency is that since adhesives are more acidic by nature, they tend to deactivate the basic amine catalyst in self- or dual-cure composites. ${ }^{10}$ The reaction of monomers and tertiary amines reduces the availability of free radicals for the polymerization reaction of the composites. ${ }^{11}$ It seems that adhesives with lower amounts of $\mathrm{pH}$ (i.e., more acidic ones) have lower bond strengths. ${ }^{12,13}$ In the present study, the adhesive used had a $\mathrm{pH}$ of 1.5 .

Many adhesives with simplified steps are now supplemented by an additional activator that contains a tertiary catalyst. However, it has been reported that the use of an activator only slightly improves the coupling with self- or dual-cure composites, or even reduces the bond strength. ${ }^{9}$

It has also been reported that single-step self-etch adhesives act as a permeable membrane, causing water to diffuse through the interface, which is one of the reasons for the premature failure of resin cement bonding to dentin. ${ }^{12,13}$

Self-etch bonding is based on two mechanisms; micromechanical bonding (monomer penetration into the interfibrillar space) and the chemical interaction between acidic functional monomers and hydroxylapatite. ${ }^{13}$ In this method, bonding is faster and less technique sensitive because, unlike procedures that require etching, it does not require moisture control, and can be used in conjunction with bulk-fill composites to reduce the chairside time. ${ }^{14}$

A push-out test is usually used to evaluate the bonding of endodontic cements to root canals. Other bond strength tests such as Shear, Tensile, Micro shear, and Micro tensile are common tests to evaluate the binding strength of resin composites. However, these tests are usually performed on a flat surface, where the C-factor (the ratio of the bonded surface to the free surface) is very low and no contraction stress is applied to the bonding surface. The advantage of the push-out test is its ability to evaluate the bond strength of a high C-factor cavity with high pressure on the bonding area. Therefore, in this study, the whole bond area is simultaneously affected by the compressive force, which allows the evaluation of shear bond strength in the cavity. ${ }^{15}$

Since the in vitro evaluation of restorative materials does not simulate the intraoral thermal changes during eating and drinking, thermocycling was performed for 10,000 cycles, which corresponds to approximately 1 year of in vivo functions. $^{8}$

The observation of failure modes after push-out bond strength tests revealed a large number of adhesive failures in all groups. In particular, all failure modes in the self- and dual-cure composite groups were adhesive failures. This might be the result of the degradation of the exposed adhesive interface by the influx of water, which leads to the hydrolysis of collagen fibers.

Differences in the type of fracture in the bulk-fill and conventional composites may indicate a lower fracture resistance in the bulk-fill group, which is probably due to its lower filler percentage. ${ }^{5}$ 
For future studies, it is recommended to use tooth-colored restorative materials from other brands and other universal adhesives with total-etch, self-etch, and selective enamel etching under clinical conditions as well as long-term followups to extend the results to clinical situations.

\section{Conclusion}

When applying universal adhesive in self-etch mode, bulkfill, and conventional light-cure composites had higher bond strength than self-and dual-cure composites. Moreover, there was no significant difference between the bond strength of bulk-fill and conventional composites.

Note

This study was based on the thesis by Dr. Mohammad Fazli.

\section{Funding}

This study was funded by the Mashhad University of Medical Sciences, Mashhad, Iran, supported the study.

\section{Conflict of Interest}

None declared.

\section{Acknowledgments}

We thank the Vice Chancellor of Research, Mashhad University of Medical Sciences, for supporting this research and the Dental Materials Research Center of Mashhad University of Medical Sciences, Mashhad, Iran, for testing the specimens (Number: 970142).

\section{References}

1 Mosharrafian S, Sharifi Z. Comparison of push-out bond strength of two bulk-fill and one conventional composite to intracanal dentin in severely damaged primary anterior teeth. J Dent (Tehran) 2016;13(03):207-214

2 Moosavi H, Hariri I, Sadr A, Thitthaweerat S, Tagami J. Effects of curing mode and moisture on nanoindentation mechanical properties and bonding of a self-adhesive resin cement to pulp chamber floor. Dent Mater 2013;29(06):708-717
3 Hughes KO, Powell KJ, Hill AE, Tantbirojn D, Versluis A. Delayed photo activation of dual-cure composites: Effect on cuspal flexure, depth-of-cure, and mechanical properties. Oper Dent 2019; 44(02):E97-E104

4 Caixeta RV, Guiraldo RD, Kaneshima EN, et al. Push-out bond strength of restorations with bulk-fill, flow, and conventional resin composites. ScientificWorldJournal 2015;2015:452976. doi: $10.1155 / 2015 / 452976$

5 Meda EM, Rached RN, Ignácio SA, Fornazari IA, Souza EM. Effect of different adhesive strategies and time on microtensile bond strength of a CAD/CAM composite to dentin. Oper Dent 2019; 44(03):262-272

6 Pereira R, Lima DANL, Giorgi MCC, Marchi GM, Aguiar FHB. Evaluation of bond strength, nanoleakage, and marginal adaptation of bulk-fill composites submitted to thermomechanical aging. J Adhes Dent 2019;21(03):255-264

7 Han SH, Park SH. Incremental and bulk-fill techniques with bulkfill resin composite in different cavity configurations. Oper Dent 2018;43(06):631-641

8 Mandava J, Vegesna DP, Ravi R, Boddeda MR, Uppalapati LV, Ghazanfaruddin MD. Microtensile bond strength of bulk-fill restorative composites to dentin. J Clin Exp Dent 2017;9(08):e1023-e1028

9 Chang J, Platt JA, Yi K, Cochran MA. Quantitative comparison of the water permeable zone among four types of dental adhesives used with a dual-cured composite. Oper Dent 2006;31(03):346-353

10 Tay FR, Suh BI, Pashley DH, Prati C, Chuang SF, Li F. Factors contributing to the incompatibility between simplified-step adhesives and self-cured or dual-cured composites. Part II. Single-bottle, total-etch adhesive. J Adhes Dent 2003;5(02):91-105

11 Cavalcanti SC, de Oliveira MT, Arrais CA, Giannini M. The effect of the presence and presentation mode of co-initiators on the microtensile bond strength of dual-cured adhesive systems used in indirect restorations. Oper Dent 2008;33(06):682-689

12 Tay FR, Pashley DH, Yiu CK, Sanares AM, Wei SH. Factors contributing to the incompatibility between simplified-step adhesives and chemically-cured or dual-cured composites. Part I. Singlestep self-etching adhesive. J Adhes Dent 2003;5(01):27-40

13 Carvalho RM, Pegoraro TA, Tay FR, Pegoraro LF, Silva NR, Pashley $\mathrm{DH}$. Adhesive permeability affects coupling of resin cements that utilise self-etching primers to dentine. J Dent 2004;32(01):55-65

14 Van Meerbeek B, De Munck J, Mattar D, Van Landuyt K, Lambrechts P. Microtensile bond strengths of an etch\&rinse and selfetch adhesive to enamel and dentin as a function of surface treatment. Oper Dent 2003;28(05):647-660

15 Segreto D, Brandt WC, Correr-Sobrinho L, Sinhoreti MA, Consani S. Influence of irradiance on the push-out bond strength of composite restorations photoactivated by LED. J Contemp Dent Pract 2008;9(02):89-96 